\title{
NLoed: A Python package for nonlinear optimal experimental design in systems biology
}

\author{
Nathan Braniff ${ }^{1}$, Taylor Pearce ${ }^{1}$, Zixuan $\mathrm{Lu}^{1}$, Michael Astwood ${ }^{1}$, William S. R. \\ Forrest $^{1}$, Cody Receno ${ }^{1}$, and Brian Ingalls ${ }^{1}$ \\ ${ }^{1}$ Department of Applied Mathematics, University of Waterloo, Waterloo, ON, N2L 3G1, Canada
}

\begin{abstract}
Motivation: Modelling in systems and synthetic biology relies on accurate parameter estimates and predictions. Accurate model calibration relies, in turn, on data, and on how well-suited the available data is to a particular modelling task. Optimal experimental design (OED) techniques can be used to identify experiments and data collection procedures that will most efficiently contribute to a given modelling objective. However, implementation of OED is limited by currently available software tools that are not well-suited for the diversity of nonlinear models and nonnormal data commonly encountered in biological research. Moreover, existing OED tools do not make use of the state-of-the-art numerical tools, resulting in inefficient computation.

Results: Here we present the NLoed software package. NLoed is an open-source Python library providing convenient access to OED methods, with particular emphasis on experimental design for systems biology research. NLoed supports a wide variety of nonlinear, multi-input/output, and dynamic models, and facilitates modelling and design of experiments over a wide variety of data types. To support OED investigations, the NLoed package implements maximum likelihood fitting and diagnostic tools, providing a comprehensive modelling workflow. NLoed offers an accessible, modular, and flexible OED tool-set suited to the wide variety of experimental scenarios encountered in systems biology research. We demonstrate NLOED's capabilities by applying it to experimental design for characterization of a bacterial optogenetic system.

Availability: NLoed is available via pip from the PyPi repository; https://pypi.org/project/nloed/. Source code, documentation and examples can be found on Github at https://github.com/ingallslab/NLoed. Contact: bingalls@uwaterloo.ca

Supplementary information: Supplementary materials are available online.
\end{abstract}

\section{Introduction}

Biological systems are heterogeneous, in terms of both their components and the interactions between them. Mathematical modelling of biological systems provides researchers with a valuable toolset for investigating this complexity. Models can be used to generate new hypotheses about extant systems or to predict properties of novel synthetic biological designs. These models are typically nonlinear, multidimensional, and dynamic, and depend on parameters that cannot be directly measured. Accurate estimation of the values of these parameters is critical: the accuracy of the parameterization determines the utility of the model predictions and the model's overall value as a tool for investigating system behaviour [Hagen et al., 2013]. Accurate parameterization of complex models often faces challenges due to the high cost of data collection. This challenge can be exacerbated by uncertainty in how to design experiments to maximize calibration accuracy.

Recent progress in experimental techniques, specifically with respect to automation and high throughput methods (reviewed by Braniff and Ingalls [2018]), reduce the barriers imposed by experimental costs. However, without complementary advances in experimental design, cheaper data collection may lead to increases in the quantity of data, but not in its quality, and so may have limited impact on model calibration accuracy. Experimental design tools are especially important for nonlinear models, for which intuition can be a poor guide [Hagen et al., 2013].

Optimal experimental design (OED) techniques were originally developed in statistics for fitting regression models accurately with minimal experimental effort [Franceschini and Macchietto, 2008]. 
These techniques have been expanded to nonlinear and dynamic models, and have seen increasing use in the past decades, including applications to biological systems, [Kreutz and Timmer, 2009, Chakrabarty et al., 2013, Braniff and Ingalls, 2018]. However, most of these methods rely on custom implementations of their specific numerical algorithms. The lack of established software tools has no doubt limited the use of OED in experimental studies, confining the use of OED techniques to research groups with specialized knowledge of OED methods (e.g. Bandara et al. [2009], Ruess et al. [2015]).

\subsection{Existing OED software tools}

A number of software tools for optimal experimental design exist, although none are specifically focused on the needs of systems biologists.

Statistical software suites such as SAS and R provide a number of optimal design packages [Groemping, 2020, Atkinson et al., 2007]. These packages are primarily aimed at regression-type models used in statistics and are of limited use to systems biologists.

Several software packages for OED have emerged from the pharmacokinetics-pharmacodynamics (PKPD) research community; examples include the PopED package from Nyberg et al. [2012] (available in MATLAB) and PFIM 3.0 from Bazzoli et al. [2010] (available in R). For a full comparison of OED tools in the PKPD field see Nyberg et al. [2015]. These PKPD tools focus on nonlinear and dynamic models. However, PKPD methods emphasize mixed-effects models (with test-subject-specific sources of variability), which can be computationally demanding and are less relevant for those working outside the PKPD field.

Software tools implementing Bayesian optimal design methods are available; a very early example is Clyde [1993]. Bayesian techniques are valuable when working with nonlinear models because they mitigate the parameter dependence of local optimal design methods. However, computational costs have often limited the complexity of models to which Bayesian methods could be applied. Recently developed techniques have made Bayesian optimal design more tractable, demonstrated by, for example, the aceBayes package in $\mathrm{R}$ by Overstall and Woods [2017]. These computational improvements have enabled Bayesian design applications to dynamic biological models [Overstall et al., 2020]. While Bayesian methods are of interest to many systems biologists, they are more computationally intensive and less user-friendly than traditional local methods. Here, we focus on local methods as a complement to Bayesian methods; we present a simple and easy-to-use implementation of the non-Bayesian OED tool-set.

\section{Methods}

In this work we present the NLoed software package, a purpose-built and user-friendly OED software toolset developed to make OED more accessible to systems biologists. The NLoed package has been released under an open-source licence; it is hosted on Github (https://github.com/ingallslab/ NLoed). The package is written in Python 3 and can be used either through a Python interpreter or in Python scripts. The NLoed package is object-oriented; it provides classes for model building and experimental design. The package classes and their associated methods can be flexibly interfaced to facilitate a variety of workflows for both real experiments and simulation studies. NLoed uses Pandas and Numpy data structures, allowing easy data exchange with other numerical packages.

In developing NLoed, we aimed to implement well-established local optimal design methods that are simple and practical, while making use of state-of-the-art numerical methods. The OED methods in NLoed rely heavily on the Fisher information matrix for design optimization. Calculation of the Fisher information relies, in turn, on accurate and rapid local parametric sensitivity computation. Moreover, design optimization requires a nonlinear programming solver. Although it is possible to estimate sensitivity and objective derivatives as finite-differences, these methods can be computationally costly and inaccurate [De Pauw and Vanrolleghem, 2006]. As an alternative, NLoed relies on CasADi [Andersson et al., 2019], a rapid prototyping package for optimal control that provides automatic differentiation functionality and a direct interface to the nonlinear programming solver IPOPT [Wächter and Biegler, 2006]. CasADi allows for rapid and accurate computation of both the Fisher information and design objective derivatives. CasADi also provides a symbolic interface for model construction, facilitating the efficient formulation of complex models. 
All models in the package take the following mathematical form:

$$
\begin{aligned}
\boldsymbol{\eta}_{i} & =\boldsymbol{f}_{i}(\boldsymbol{x}, \boldsymbol{\theta}), \\
Y_{i} & \sim p_{i}\left(y_{i} \mid \boldsymbol{\eta}_{i}\right) .
\end{aligned}
$$

Here the vector $\boldsymbol{x}$ captures the model inputs while the vector $\boldsymbol{\theta}$ consists of the model parameters. The index $i$ is used to distinguish separate observations. For each value of $i$, the function $\boldsymbol{f}_{i}(\cdot)$ maps model inputs and parameter values to the observation's sampling statistics, collected in vector $\boldsymbol{\eta}_{i}$. The sampling statistics characterize the distribution that describes a given observation. For example, for a normally distributed observation variable, the sampling statistics are the mean and variance $\left(\boldsymbol{\eta}=\left(\mu, \sigma^{2}\right)=(\right.$ mean, variance $\left.)\right)$, which completely characterize the distribution from which the observation is drawn. (Recall that in statistical modelling, observations are treated as realizations of a random variable that is established by the model; the function $\boldsymbol{f}(\cdot)$ describes that random variables in terms of the model inputs and parameters.) In the NLoed package, the function $\boldsymbol{f}(\cdot)$ is implemented using CasADi's symbolics, which can consist of analytic expressions or numerical algorithms. The number of observations and inputs an NLoed model can accept is constrained only by the computational cost of computing optimal designs. NLoed can be used to optimize designs for multi-input/output models, including multi-state dynamic systems.

Equation 2 specifies each observation, $Y_{i}$, as drawn from the probability distribution specified by the sampling statistics $\boldsymbol{\eta}_{i}$. The notation $p_{i}\left(y_{i} \mid \boldsymbol{\eta}_{\boldsymbol{i}}\right)$ emphasizes the conditioning on the sampling statistics. Using this model formulation, NLoed accommodates experimental observations that are non-normally distributed, including counts and strictly positive data. The user can specify a specific distribution type for each observation variable according to the experimental scenario. Supported distributions include Normal, Poisson, Binomial, Bernoulli, Lognormal, Exponential, and Gamma. NLoed is thus applicable to a wide variety of experimental scenarios (e.g. involving both gene expression and plate counts) and modelling frameworks (e.g. deterministic approximations of stochastic models via moment closure [Lakatos et al., 2015] or the linear noise approximation [Elf and Ehrenberg, 2003]). Note that an observation $Y_{i}$ corresponds to a measurement from a specific observation channel (e.g. concentration, light intensity, optical density, etc.) at a specific, pre-specified time-point. (So, e.g., a measurement repeated at two different time-points corresponds to two different observations $Y_{i}$.)

For a given system (1-2), an experimental design in NLoed is defined by a pair of sets $\mathcal{D}=\{\boldsymbol{X}, \boldsymbol{\Xi}\}$ characterizing inputs and observation replicate counts, as follows. Here $\boldsymbol{X}$ is the set of input vectors, $\boldsymbol{x}_{j}$, that describe the set of experiments to be executed, i.e. the set of experimental conditions to be observed. The input vector can encode the numerical settings of treatments such as chemical concentrations or light intensity, or environmental conditions such as temperature or media composition. Categorical perturbations, such as strain or treatment type, can be encoded as binary entries. Time-varying inputs are implemented as piece-wise constants by first subdividing the experimental time window and then specifying a constant value over each sub-interval.

The system specification (1-2) defines the collection of possible observations $Y_{i}$. The set $\Xi$ contains the allocation of replicates: observable $Y_{i}$ is to be assessed $\xi_{i, j}$ times under the $j$ th input setting. These are (non-negative) integer valued counts. However to improve numerical tractability, NLoed relaxes this integer constraint so that $\xi_{i, j}$ correspond to non-negative real-valued weights. These real-value allocations are then rounded to discrete values after an optimal solution is found. We refer to a design with real-valued replicate allocations as relaxed, while a design with integer-valued allocations is called exact.

Design optimization problems in NLoed have the following general form:

$$
\begin{gathered}
\max _{\mathcal{D}} \Psi(\mathcal{I}(\mathcal{D}, \overline{\boldsymbol{\theta}})), \text { where } \\
\mathcal{I}(\mathcal{D}, \overline{\boldsymbol{\theta}})=\sum_{j}^{N} \sum_{i}^{M} \xi_{i, j} \mathcal{I}_{i}\left(\boldsymbol{x}_{j}, \overline{\boldsymbol{\theta}}\right)
\end{gathered}
$$

Here $\mathcal{I}(\mathcal{D}, \overline{\boldsymbol{\theta}})$ is the expected Fisher information matrix for experimental design $\mathcal{D}$, evaluated at the nominal parameter vector $\overline{\boldsymbol{\theta}}$. The Fisher information appears in the objective because it is asymptotically related to the expected variability of estimates of the model parameters determined by maximum likelihood estimation [Fedorov and Leonov, 2013]. The Fisher information therefore serves as a useful metric for design utility. The objective function, $\Psi(\cdot)$, maps the Fisher information matrix to a scalar 
objective. By default NLoed uses the determinant of the inverse of the Fisher information matrix as its objective; this results in a D-optimal design which minimize the expected confidence volume of the parameter estimates. The integer $N$ is the number of unique input vectors considered in the design and $M$ is the number of observables (i.e. output channel-timepoint pairs). The relaxed replicate allocations, $\xi_{i, j}$ are constrained to sum to one: $1=\sum_{i}^{M} \sum_{j}^{N} \xi_{i, j}$.

The expected Fisher information for a design is determined as the sum of the individual Fisher information matrices, $\mathcal{I}_{i}\left(\boldsymbol{x}_{j}, \overline{\boldsymbol{\theta}}\right)$, for each input vector, $\boldsymbol{x}_{j}$ and observation, $y_{i}$. The individual Fisher information matrices are defined as

$$
\mathcal{I}_{i}\left(\boldsymbol{x}_{j}, \overline{\boldsymbol{\theta}}\right)=E_{y_{i}}\left[\frac{\partial \log \left(p_{i}\left(y_{i} \mid \boldsymbol{f}_{i}\left(\boldsymbol{x}_{j}, \overline{\boldsymbol{\theta}}\right)\right)\right)}{\partial \boldsymbol{\theta}} \cdot \frac{\partial \log \left(p_{i}\left(y_{i} \mid \boldsymbol{f}_{i}\left(\boldsymbol{x}_{j}, \overline{\boldsymbol{\theta}}\right)\right)\right)^{T}}{\partial \boldsymbol{\theta}}\right] .
$$

This is the expected value of the square of the parametric sensitivities of the log-likelihood (i.e. variance of the score vector) [Fedorov and Leonov, 2013]. NLoed uses a chain rule decomposition to simplify the computation of the Fisher information, as follows [Fedorov and Leonov, 2013]. From the definition in Equation 2 we have that $\boldsymbol{\eta}_{i}=\boldsymbol{f}_{i}(\boldsymbol{x}, \boldsymbol{\theta})$ and therefore the log-likelihood sensitivity vector can be decomposed as:

$$
\begin{aligned}
\frac{\partial \log \left(p_{i}\left(y \mid \boldsymbol{\eta}_{i, j}\right)\right)}{\partial \boldsymbol{\theta}} & =\frac{\partial \log \left(p_{i}\left(y \mid \boldsymbol{\eta}_{i, j}\right)\right)}{\partial \boldsymbol{\eta}_{i, j}} \cdot \frac{\partial \boldsymbol{\eta}_{i, j}}{\partial \boldsymbol{\theta}} \\
& =\frac{\partial \log \left(p_{i}\left(y \mid \boldsymbol{\eta}_{i, j}\right)\right)}{\partial \boldsymbol{\eta}_{i, j}} \cdot \frac{\partial \boldsymbol{f}_{i}\left(\boldsymbol{x}_{j}, \boldsymbol{\theta}\right)}{\partial \boldsymbol{\theta}}
\end{aligned}
$$

The term $\partial \boldsymbol{f}_{i}\left(\boldsymbol{x}_{j}, \boldsymbol{\theta}\right) / \partial \boldsymbol{\theta}$ is the parametric sensitivity of the sampling statistics. This sensitivity can be determined via automatic differentiation of the user-provided model function $\boldsymbol{f}(\cdot)$. The term $\partial \log \left(p_{i}\left(y \mid \boldsymbol{\eta}_{i, j}\right) / \partial \boldsymbol{\eta}_{i, j}\right.$ and the expectation can be subsumed into the matrix $\Phi_{i}\left(\boldsymbol{x}_{j}, \overline{\boldsymbol{\theta}}\right)$, defined as:

$$
\Phi_{i}\left(\boldsymbol{x}_{j}, \overline{\boldsymbol{\theta}}\right)=E_{Y}\left[\frac{\partial \log \left(p_{i}\left(y \mid \boldsymbol{\eta}_{i, j}\right)\right.}{\partial \boldsymbol{\eta}_{i, j}} \cdot \frac{\partial \log \left(p_{i}\left(y \mid \boldsymbol{\eta}_{i, j}\right)^{T}\right.}{\partial \boldsymbol{\eta}_{i, j}}\right] .
$$

which can be computed analytically from the user-provided function $\boldsymbol{f}(\cdot)$ for the observation distributions supported by NLoed [Fedorov and Leonov, 2013]. The Fisher information for each input and observation in a design can then be evaluated as

$$
\mathcal{I}_{i}\left(\boldsymbol{x}_{j}, \overline{\boldsymbol{\theta}}\right)=\frac{\partial \boldsymbol{f}_{i}\left(\boldsymbol{x}_{j}, \overline{\boldsymbol{\theta}}\right)}{\partial \boldsymbol{\theta}} \Phi_{i}\left(\boldsymbol{x}_{j}, \overline{\boldsymbol{\theta}}\right) \frac{\partial \boldsymbol{f}_{i}\left(\boldsymbol{x}_{j}, \overline{\boldsymbol{\theta}}\right)^{T}}{\partial \boldsymbol{\theta}} .
$$

For numerical tractability, the design optimization problem can be further simplified in a variety of ways, depending on how the candidate input vector $\boldsymbol{x}_{j}$ and replicate allocations, $\xi_{i, j}$ are encoded as optimization variables in the nonlinear programming solver. NLoed offers the users ample flexibility in how the optimization problem is posed via various user-controlled settings.

In addition to design optimization, NLoed includes several model-building and diagnostic tools, including methods for model fitting, sensitivity analysis, model simulation, data sampling and design evaluation. These auxiliary tools are complementary to NLoed's primary OED functionality. They facilitate incorporation of NLoed into a complete model building and experimental workflow that aligns with the user's optimal design objectives.

\section{Implementation}

The NLoed library is built around two core classes: the Model class, and the Design class. The Model class captures all of the mathematical information specified in the model framework in Equations (12). The Model class also provides methods for model calibration and simulation and for evaluating a given design's performance on the model instance. The Design class accepts Model instances, as well as other design information, and then implements and solves the design optimization problem. The Design class also provides methods for rounding a relaxed design to an exact design with a specified total sample budget. 


\subsection{Model Building}

As an example, we consider the CcaS/CcaR optogenetic system described in Schmidl et al. [2014]. (This system was previously characterized in Olson et al. [2017]. A closely related system was characterized in Olson et al. [2014] by a model similar to the one presented below.) We focus on the system's steady-state response to pulse-width modulated (PWM) green light. (Application to a complementary dynamic model is illustrated in the supplement.) We describe the steady-state response by a Hill function model with normally distributed heteroskedastic observation errors:

$$
\begin{aligned}
\mu & =f(x, \theta)=\alpha_{o}+\alpha \frac{x^{n}}{K^{n}+x^{n}}, \\
Y & \sim N\left(\mu, s^{2} \mu^{2}\right)
\end{aligned}
$$

Here the single input $x$ is the green light level delivered during growth of the culture (as a percentage of maximal light level). The single independent sampling statistic $\eta=\mu$ is the observation mean. The single observable $Y$, assumed to be normally distributed, is the steady-state mean GFP expression of a batch culture (see the supplement for details). The components of the unknown model parameter vector $\theta=\left(\alpha_{o}, \alpha, n, K\right)$ characterize basal expression, maximal induced expression, sensitivity (Hill coefficient), and half-maximal input, respectively. The variance of the GFP observations is assumed to be proportional to the square of the mean, with $s$ serving as the proportionality constant. We were not interested in optimizing designs for estimates of $s$; its value was therefore fixed based on initial data (see the supplement for details).

To define a model in NLoed we first specify an expression for the function $\boldsymbol{f}(\cdot)$ mapping inputs to sampling statistics using CasADi's symbolic types. Code listing 1 shows this process for the model in Equation (9).

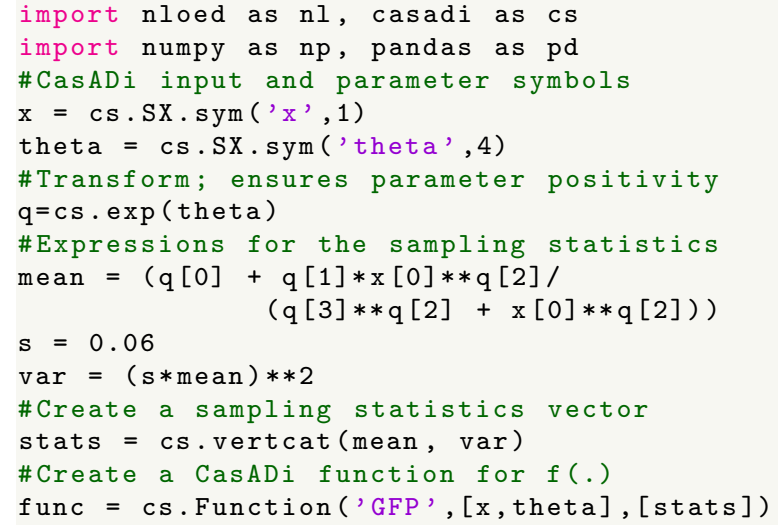

Listing 1: Code for creating a CasADi function for the CcaS/CcaR model's sampling statistics. The value of $s$ set here was estimated from initial data (details in the supplement).

In Listing 1, a log transformation of the original parameters from Equation (9) is used to ensure the parameter values remain positive during fitting. In the final line of Listing 1, a CasADi function, func, is generated to implement $\boldsymbol{f}(\cdot)$ from equation (9). The user can then call the NLoed Model class constructor to create the NLoed model instance; this is shown in Listing 2.

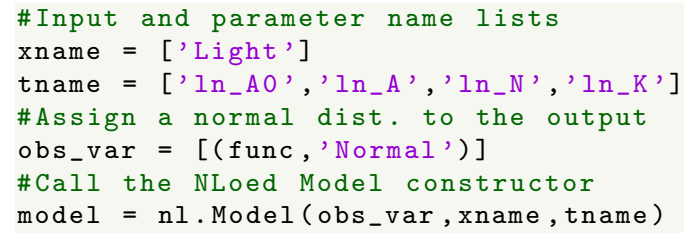

Listing 2: Code for creating an NLoed Model class instance for the CcaS/CcaR model.

For the CcaS/CcaR case study, we conducted initial experiments observing, in triplicate, individual batch cultures' mean GFP expression in steady-state under $0 \%, 1.5 \%, 6 \%, 25 \%$, and $100 \%$ of the maximal green light level (15 observations in total, details in the supplement). This preliminary dataset was used to generate initial parameter estimates, from which NLoed's locally optimal designs 
could be determined. Listing 3 details the use of the Model class's fit() method in generating initial parameter estimates of $\alpha_{o}=552.8$ a.u., $\alpha=9493.2$ a.u., $K=8.5 \%$ and, $n=2.4$. The variance proportionality constant was estimated independently as $s=0.06$ (see supplement for details).

Beyond fitting, Model class instances can perform a number of model development tasks via available class methods:

Fitting diagnostics: In addition to implementing a maximum likelihood fitting algorithm (as in Listing 3), the fit() method can generate profile likelihood-based confidence intervals for the parameter estimates and can plot visual diagnostics of parameter identifiability such as confidence contours, likelihood profiles and profile trace projections [Bates and Watts, 1988].

Model predictions: The predict() method allows the user to generate predictions of the means of the model's observable outputs for a given input setting. This method can also generate prediction uncertainty intervals to quantify the effects of parameter uncertainty on predictions and can perform local parametric sensitivity analysis.

Design evaluation: The evaluate() method allows the user to evaluate candidate experimental designs with respect to the given model using interpretable metrics such as the expected covariance, bias and mean squared error of the parameter estimates. The evaluate() method can use asymptotic or Monte Carlo-based computation to determine the design evaluation metrics.

Data simulation: The sample() method complements the predict() method by simulating random experimental observations from a given experimental design, allowing the user to generate sample data for simulation studies.

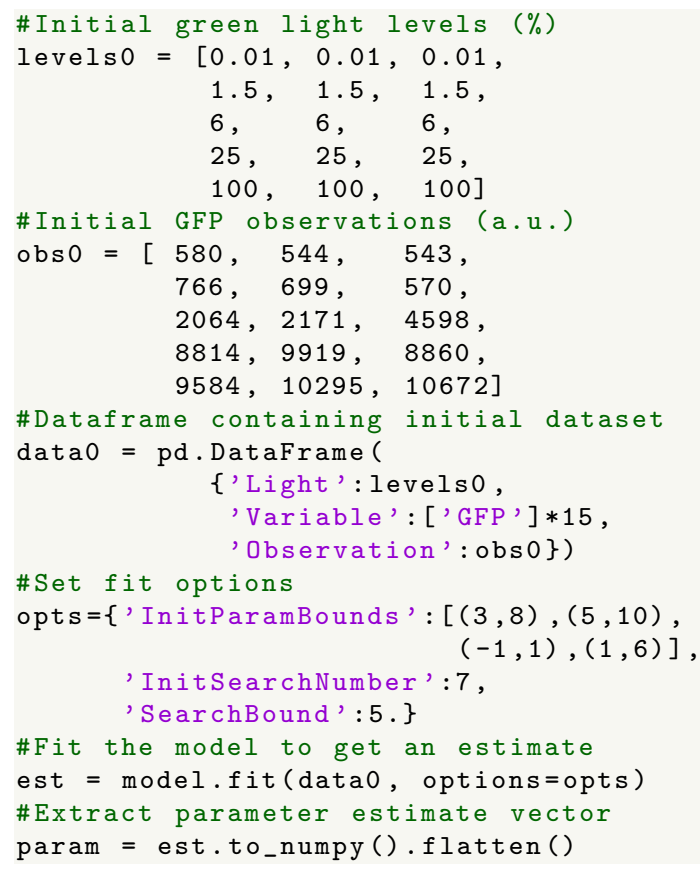

Listing 3: Code for fitting the NLoed Model class instance of the CcaS/CcaR model to an initial dataset.

\subsection{Optimal Experimental Design}

To generate an optimal design for the CcaS/CcaR model, we passed the previously created Model class instance to the Design constructor (Listing 4). The call to the Design constructor shown in Listing 4 includes specification of the model object, input constraints, design objective, and the nominal parameter values around which the design is to be optimized. In the first lines of Listing 4 the inp dictionary is used to define the input constraints. This dictionary specifies the bounds and the number of unique levels of the green light input that are to be allowed during the design optimization. In 
this case the light level is bounded between $0.01 \%$ and $100 \%$ and designs consisting of at most four unique levels are considered. NLoed provides a variety of options for how the input levels are handled numerically and which constraints are applied; see the documentation on Github for further details.

The nominal parameter values are needed in the Design constructor call because NLoed uses the expected Fisher information - a local asymptotic approximation - to compute the design objective. The optimality of the resulting design is therefore dependent on how close the nominal values are to the unknown true parameters. It can thus be risky to optimize the design for an entire experiment based on highly uncertain nominal parameter values. This risk can be mitigated by performing a series of sequentially optimized experiments [Atkinson et al., 2007]. In sequential design, rather than using an uncertain nominal parameter set to allocate all observations in a study, the experimenter subdivides their planned experiment into a series of sub-experiments. In this scenario, the experimental design generated for the first sub-experiment may be sub-optimal, but even a sub-optimal experiment yields additional data. This data increases the the sample size - and as parameter estimation accuracy is generally expected to increase with the square root of the sample size [Fedorov and Leonov, 2013] - each additional sub-experiment will on average yield parameter estimates that are nearer to the unknown true value. Therefore, by optimizing each subexperiment with respect to the current best parameter estimate, the optimality of the combined dataset with respect to the unknown true parameter values is expected to improve. To demonstrate this process, in Listing 4 the parameter estimates from the preliminary experiment are used as the nominal values for the design of an optimized experimental run on the CcaS/CcaR system.

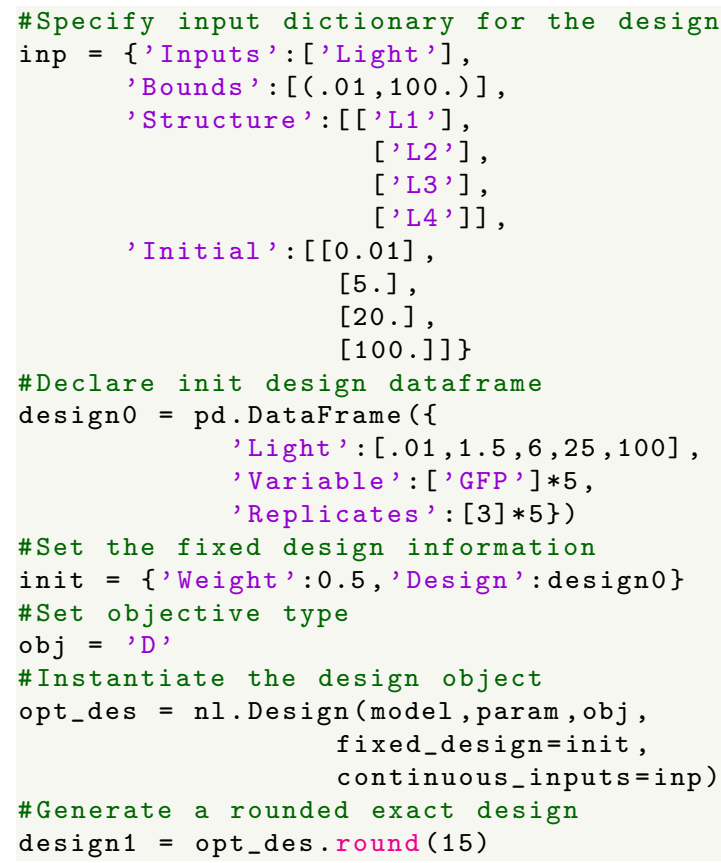

Listing 4: Code demonstrating the creation and optimization of a Design object for the CcaS/CcaR model.

Using the parameter estimates from a previous (sub-)experiment for design optimization will improve the expected performance of the resulting design. However, even greater efficiency can be achieved by conditioning the design optimization directly on past data as well. A subsequent experiment can be most efficiently selected if it is optimized to complement the previously-gathered observations with respect to the objective, rather than disregarding the information already available. This approach results in a conditionally optimal design at each iteration of a sequential design procedure: each design is conditionally optimal on all of the past data in the sense that the new design assumes that the past observations will be included in any subsequent parameter estimation. This conditional optimization is shown in Listing 4 where the initial design, design0, is passed into the Design constructor via the init field. By passing in the initial design, NLoed will select a new design that best complements the initial design in order to optimize the objective.

When the Design class is instantiated near the end of Listing 4, NLoed runs an IPOPT call to 
Table 1: An optimal design for the CcaS/CcaR model.

\begin{tabular}{ll} 
Green Light Input & $\begin{array}{l}\text { Number of Replicates } \\
\boldsymbol{x}_{j}\end{array}$ \\
\hline $\boldsymbol{x}_{1}=0.01 \%$ & 4 \\
$\boldsymbol{x}_{2}=2.8 \%$ & 5 \\
$\boldsymbol{x}_{3}=8.1 \%$ & 4 \\
$\boldsymbol{x}_{4}=100.0 \%$ & 2
\end{tabular}

generate an optimal relaxed design, in which the optimal allocation of replicates assumes a continuum of replicate allocation weights $\Xi$. To produce a design that can be implemented, the last line of listing 4 uses the round () method to generate a usable design with a total of 15 replicates (over all observations, mirroring the design of the preliminary experiment). The resulting optimized exact design contained in variable design1 is shown in Table 1. Note that the optimal design, perhaps non-intuitively, focuses on the lowest part of the input range.

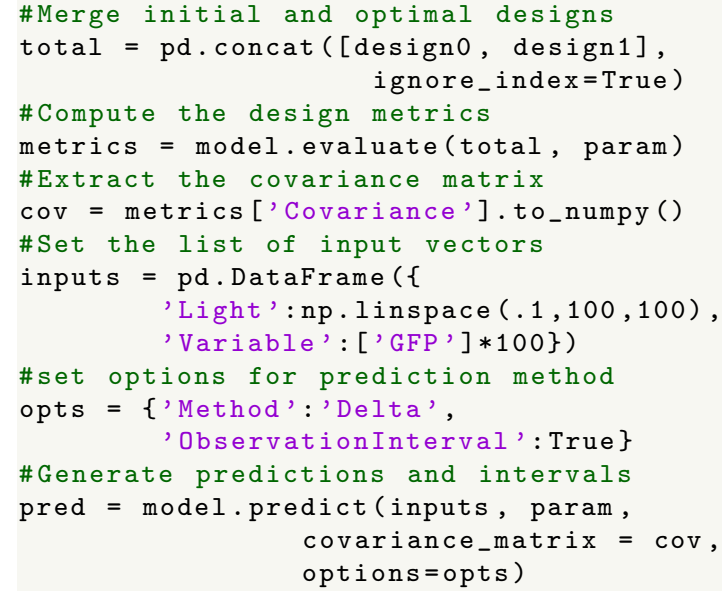

Listing 5: Code for generating design evaluation metrics and model predictions.

We then executed the optimal design and re-fit the model to the combined data (preliminary and follow-up optimal). The updated parameter estimates are $\alpha_{o}=525.6$ a.u., $\alpha=9876.1$ a.u., $K=10.4 \%$ and, $n=2.3$. To compute the size of each parameter's approximate $95 \%$ confidence interval, we used the Model class's evaluate() method (Listing 5 , full code in the supplement). In Listing 5 , the evaluate() method produces a parameter covariance matrix for a combined design including both the initial design, design0, and the optimal design, design1. The covariance matrix is then used to compute the asymptotic confidence intervals. For comparison, we generated asymptotic confidence intervals for two other cases: (i) the preliminary experiment alone, and (ii) the preliminary experiment followed by a replicate of the preliminary experiment (code provided in supplement). (The replicate initial design provides a controlled comparison for the increased sample size). Figure 1 shows the asymptotic confidence intervals for all three cases. These results show improved precision of the parameter estimates using the optimized design rather than simply replicating the original design. These improvements are primarily accrued in the two nonlinear parameter $K$ and $n$, where the optimal design noticeably outperforms direct replication.

The impact of the experimental design on model performance can be visualized by the effects of uncertainty on model predictions. NLoed produces the necessary data through the Model class's predict () method. After generating the covariance matrix in Listing 5 , the matrix is then passed to the predict() method to generate model predictions and a $95 \%$ uncertainty interval for the observations. This interval accounts for observation variability and the parameter uncertainty resulting from the overall design. Figure 2 shows a plot of the model predictions and observation interval along with the initial and optimal data. 


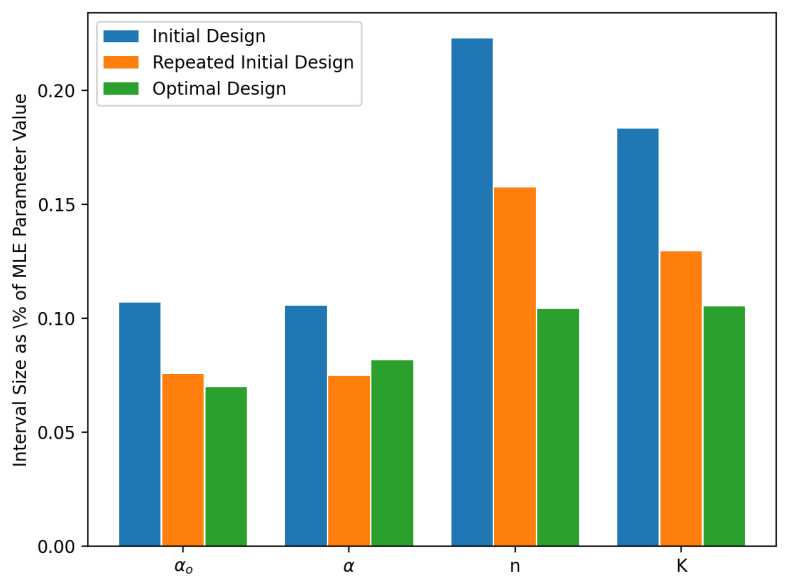

Figure 1: A comparison of $95 \%$ confidence interval sizes, expressed as a percentage of the parameter estimate values, between various combinations of the initial and optimal designs.

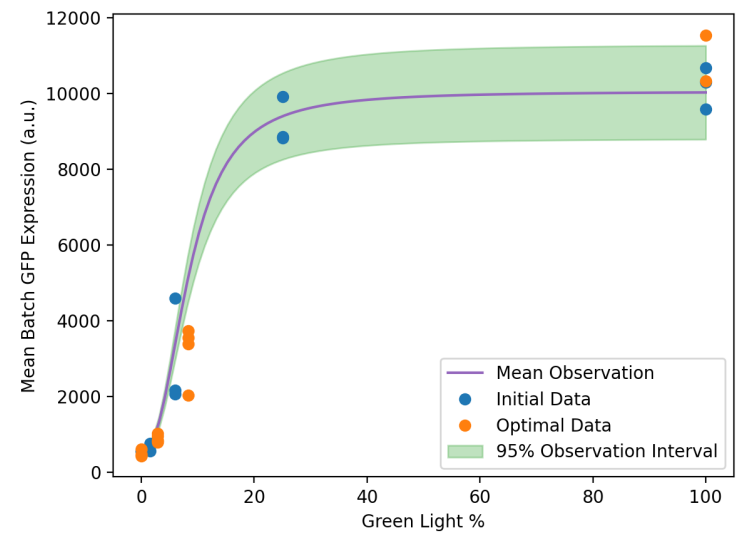

Figure 2: Model predictions and 95\% observation interval for the CcaS/CcaR model. Also shown are the initial and optimal data used for fitting the model.

\section{Discussion}

Optimal experimental design (OED) consists of a well established set of methodologies with a long history of success. However, it remains a challenge to translate OED methods into practice, especially for evolving experimental disciplines such as systems biology. NLoed aims to make OED more accessible to systems biologists by providing OED methods in an easy-to-use and open-source package. By combining OED methods with general model building and diagnostic algorithms, NLoed provides a complete modelling workflow. NLoed's use of state-of-the-art automatic differentiation tools, via CasADi, improves performance and makes the package easily extensible.

Currently, NLoed only implements local asymptotic optimization criteria for parameter accuracy. NLoed is therefore best suited to designing large, iterative characterization experiments. In these cases, when the sample size is large, the asymptotic and local approximations are expected to perform well, especially when used for sequential design. NLoed's focus on parameter accuracy also means it is especially useful for precise modelling of well understood natural system or synthetically engineered systems where the model structure is reasonably well determined: it is better suited to precision characterization of well studied systems as opposed to early investigation of novel ones.

In future releases we hope to expand NLoed's capabilities, including new design methods such as pseudo-Bayesian techniques for addressing model and parameter uncertainty [Schenkendorf et al., 2009]. This will improve NLoed's ability to accommodate uncertainty, especially for early experimental 
work on novel systems. Regardless, in its current form, NLoed can make it easier for experimentalists to more efficiently allocate laboratory resources while also providing theoretical groups with tools to study the effects of experimental design on model identifiability.

\section{Acknowledgements}

The plasmids used in this work were generously provided by Jeffery Tabor's group at Rice University, from the work Schmidl et al. [2014] .

\section{Funding}

This work was supported by a Discovery Grant from Canada's Natural Sciences and Engineering Research Council (NSERC).

\section{References}

David R Hagen, Jacob K White, and Bruce Tidor. Convergence in parameters and predictions using computational experimental design. Interface Focus, 3(4):20130008, 2013.

Nathan Braniff and Brian Ingalls. New opportunities for optimal design of dynamic experiments in systems and synthetic biology. Current Opinion in Systems Biology, 2018.

Gaia Franceschini and Sandro Macchietto. Model-based design of experiments for parameter precision: State of the art. Chemical Engineering Science, 63(19):4846-4872, 2008.

Clemens Kreutz and Jens Timmer. Systems biology: experimental design. The FEBS Journal, 276 (4):923-942, 2009.

Ankush Chakrabarty, Gregery T Buzzard, and Ann E Rundell. Model-based design of experiments for cellular processes. Wiley Interdisciplinary Reviews: Systems Biology and Medicine, 5(2):181-203, 2013.

Samuel Bandara, Johannes P Schlöder, Roland Eils, Hans Georg Bock, and Tobias Meyer. Optimal experimental design for parameter estimation of a cell signaling model. PLoS Computational Biology, 5(11):e1000558, 2009.

Jakob Ruess, Francesca Parise, Andreas Milias-Argeitis, Mustafa Khammash, and John Lygeros. Iterative experiment design guides the characterization of a light-inducible gene expression circuit. Proceedings of the National Academy of Sciences USA, 112(26):8148-8153, 2015.

Ulrike Groemping. CRAN task view: Design of experiments (DoE) \& analysis of experimental data, 2020. URL https://cran.r-project.org/web/views/ExperimentalDesign.html.

Anthony Atkinson, Alexander Donev, Randall Tobias, et al. Optimum experimental designs, with SAS, volume 34. Oxford University Press, 2007.

Joakim Nyberg, Sebastian Ueckert, Eric A Strömberg, Stefanie Hennig, Mats O Karlsson, and Andrew C Hooker. PopED: an extended, parallelized, nonlinear mixed effects models optimal design tool. Computer methods and programs in biomedicine, 108(2):789-805, 2012.

Caroline Bazzoli, Sylvie Retout, and France Mentré. Design evaluation and optimisation in multiple response nonlinear mixed effect models: PFIM 3.0. Computer methods and programs in biomedicine, 98(1):55-65, 2010.

Joakim Nyberg, Caroline Bazzoli, Kay Ogungbenro, Alexander Aliev, Sergei Leonov, Stephen Duffull, Andrew C Hooker, and France Mentré. Methods and software tools for design evaluation in population pharmacokinetics-pharmacodynamics studies. British journal of clinical pharmacology, 79(1): 6-17, 2015.

Merlise A Clyde. An object-oriented system for bayesian nonlinear design using xlisp-stat. Technical report, University of Minnesota, 1993. 
Antony M Overstall and David C Woods. Bayesian design of experiments using approximate coordinate exchange. Technometrics, 59(4):458-470, 2017.

Antony M Overstall, David C Woods, and Ben M Parker. Bayesian optimal design for ordinary differential equation models with application in biological science. Journal of the American Statistical Association, pages 1-16, 2020.

Dirk JW De Pauw and Peter A Vanrolleghem. Avoiding the finite difference sensitivity analysis deathtrap by using the complex-step derivative approximation technique. International Congress on Environmental Modelling and Software., 24, 2006.

Joel A E Andersson, Joris Gillis, Greg Horn, James B Rawlings, and Moritz Diehl. CasADi - A software framework for nonlinear optimization and optimal control. Mathematical Programming Computation, 11(1):1-36, 2019. doi: 10.1007/s12532-018-0139-4.

Andreas Wächter and Lorenz T Biegler. On the implementation of an interior-point filter line-search algorithm for large-scale nonlinear programming. Mathematical Programming, 106(1):25-57, 2006.

Eszter Lakatos, Angelique Ale, Paul DW Kirk, and Michael PH Stumpf. Multivariate moment closure techniques for stochastic kinetic models. The Journal of chemical physics, 143(9):094107, 2015.

Johan Elf and Måns Ehrenberg. Fast evaluation of fluctuations in biochemical networks with the linear noise approximation. Genome research, 13(11):2475-2484, 2003.

Valerii V Fedorov and Sergei L Leonov. Optimal design for nonlinear response models. CRC Press, 2013.

Sebastian R Schmidl, Ravi U Sheth, Andrew Wu, and Jeffrey J Tabor. Refactoring and optimization of light-switchable escherichia coli two-component systems. ACS synthetic biology, 3(11):820-831, 2014.

Evan J Olson, Constantine N Tzouanas, and Jeffrey J Tabor. A photoconversion model for full spectral programming and multiplexing of optogenetic systems. Molecular systems biology, 13(4):926, 2017.

Evan J Olson, Lucas A Hartsough, Brian P Landry, Raghav Shroff, and Jeffrey J Tabor. Characterizing bacterial gene circuit dynamics with optically programmed gene expression signals. Nature Methods, 11(4):449-455, 2014.

Douglas M Bates and Donald G Watts. Nonlinear regression analysis and its applications, volume 2. Wiley New York, 1988.

R Schenkendorf, A Kremling, and M Mangold. Optimal experimental design with the sigma point method. IET systems biology, 3(1):10-23, 2009. 